\title{
Statistical mechanics of the triple contact line
}

\author{
Michel Y. Louge* \\ Sibley School of Mechanical and Aerospace Engineering, Cornell University, Ithaca, New York 14853, USA \\ (Received 15 September 2016; revised manuscript received 19 December 2016; published 31 March 2017)
}

\begin{abstract}
We outline a statistical mechanics of the triple gas-solid-liquid contact line on a rough plane. The analysis regards the neighborhood of the line as a solid dotted with cavities. It adopts the simplest mean-field statistical mechanics, in which each cavity is either full or empty, while being connected to near neighbors by thin necks. The theory predicts equilibrium angles for advance and recession in terms of the Young contact angle and the joint statistical distribution of two quantifiable geometrical parameters representing specific neck cross-section and specific cavity opening. It attributes contact angle hysteresis to first-order phase transitions among adjacent cavities, as they collectively imbibe or reject liquid. It also calculates the potential energy barriers that hysteresis erects against overcoming contact line pinning. By determining whether the phase transitions can release latent energy, this $a b$ initio analysis distinguishes six regimes, including two metastable recession states. We compare predictions with data for superhydrophobia on microscopic rods; for hysteresis in the "Wenzel state"; and for variations of the advancing contact angle with surface energies of the liquid.
\end{abstract}

DOI: 10.1103/PhysRevE.95.032804

\section{INTRODUCTION}

When liquid advances on a dry solid surface, the triple gas-solid-liquid contact line adopts a greater contact angle $\theta_{a}$ than when it recedes at the angle $\theta_{r}, 0<\theta_{r} \leq \theta_{a}<\pi$. The measure $\left(\cos \theta_{r}-\cos \theta_{a}\right) \geq 0$ of this hysteresis typically widens with greater surface roughness [1-10]. In addition, the line can "pin"; i.e., it no longer musters enough energy to move. For example, a sessile drop laid on a solid plane under gravity stays put until the plane is sufficiently inclined, with a smaller contact angle at the point of highest elevation than at its lowest [11-14]. Because this difference in $\theta$ exists without any visible fluid motion, a thermodynamic approach that does not account for time, but instead recognizes the direction of any quasistatic change in state variables, should be sufficient to describe this hysteretic phenomenon.

Inspired by our recent work on statistical mechanics of unsaturated porous media [15], we develop here a similar $a b$ initio theory for the contact line. The analysis predicts equilibrium contact angles of advance and recession, and it suggests how microscopic geometrical features of the solid surface determine the hysteresis. De Coninck [16] also invoked statistical mechanics to describe this phenomenon. We now revisit this approach to derive explicitly how geometry of the rough surface gives rise to six distinct regimes of contact line behavior, and we identify these regimes in experimental data.

\section{STATISTICAL MECHANICS OF THE CONTACT LINE}

We describe a microscopically rough flat plate as a collection of unit cells each consisting of a cavity of surface area $a_{p}$ with opening of area $a_{o}$ and volume $v_{p}$ surrounded by a

\footnotetext{
*Michel.Louge@ cornell.edu

Published by the American Physical Society under the terms of the Creative Commons Attribution 3.0 License. Further distribution of this work must maintain attribution to the author(s) and the published article's title, journal citation, and DOI.
}

flat solid surface of area $a_{s}$ (Fig. 1). We examine a large number of unit cells in the neighborhood of a triple liquid-gas-solid contact resting at a contact angle $\theta$. We denote by $\gamma_{g s}, \gamma_{s \ell}$, and $\gamma_{\ell g}$ the respective surface energies of gas-solid, solid-liquid, and liquid-gas interfaces.

The probability that the line has overcome a cell in this neighborhood is $0 \leq \chi \leq 1$. As $\mathrm{Xu}$ and Louge assumed [15], the neighborhood has "mesoscopic" size larger than the microscopic roughness, but smaller than the macroscopic dimension of the whole system, which, in the case of the contact line, scales with the inverse of the local gas-liquid interface curvature $\kappa$. As Xu and Louge [15] estimated, the mesoscopic domain involves up to the $m$ th nearest neighbor of a unit cell,

$$
m \sim-\left[\frac{S}{\ln S}+\frac{1-S}{\ln (1-S)}\right]
$$

where $0 \leq S \leq 1$ is the mean saturation of cavities in wetting liquid. While this equation is instructive to estimate how many surface cavities are involved in a mesoscopic neighborhood of the contact line, the size of this domain does not explicitly appear in the analysis that follows. Therefore, accuracy of this estimate is not crucial.

We adopt an Ising model whereby the cavity of an individual unit cell can only be full or empty of liquid. We define a state variable $\sigma=+1$ for an empty cavity and $\sigma=-1$ for a filled one. The first step in deriving the statistical mechanics is to calculate how much energy must be supplied to the unit cell to change its state from $-\sigma$ to $+\sigma$. To that end, without loss of generality, we consider the process of filling cavities like that sketched in Fig. 1.

This process involves four kinds of energy input to the unit cell. The first is the work $W$ across the volume $v_{p}$ of the pressure difference $\left(p_{\ell}-p_{g}\right)=\kappa \gamma_{\ell g}$ transmitted from the nearby gas-liquid interface of the macroscopic liquid drop,

$$
W=+\kappa \gamma_{\ell g} v_{p}
$$



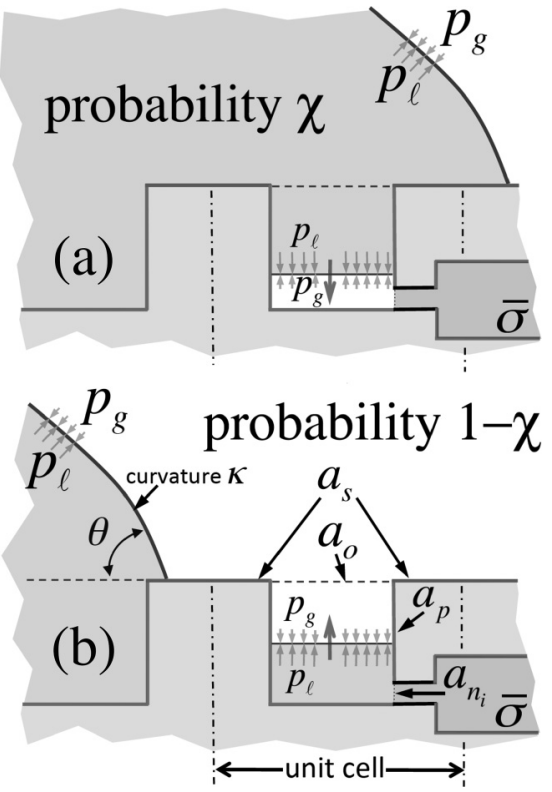

FIG. 1. Sketch of an individual unit cell consisting of a solid surface of area $a_{s}$, and a cavity of opening area $a_{o}$ and surface area $a_{p}$. The cavity is connected to its neighbors through several "necks" of cross-section $a_{n_{i}}$. (a) The cavity fills after the liquid drop and its contact line have overcome it, with probability $\chi$. (b) The cavity fills through adjacent necks before drop and line have overcome it, with probability $(1-\chi)$.

The second is the surface energy needed to substitute gas with liquid on the cavity wall of area $a_{p}$,

$$
\Gamma_{p}=\left(\gamma_{s \ell}-\gamma_{g s}\right) a_{p}=-a_{p} \gamma_{\ell g} \cos \theta_{e}
$$

where

$$
\cos \theta_{e} \equiv\left(\gamma_{g s}-\gamma_{s \ell}\right) / \gamma_{\ell g}
$$

is the "Young" equilibrium contact angle cosine on the same solid without any cavities or defects.

The third is the energy needed to create (or remove) a gasliquid interface across the cavity opening of area $a_{o}$. For this process, we distinguish whether the unit cell has already been overtaken by the contact line [Fig. 1(a), with probability $\chi$ ], or not [Fig. 1(b)]. If it has, adding liquid to the cavity produces the "exothermic" destruction of a gas-liquid interface liberating the energy $-a_{o} \gamma_{\ell g}$; if it has not, adding liquid implies the "endothermic" creation of a similar interface requiring $+a_{o} \gamma_{\ell g}$. On average over several unit cell of similar geometry, the process is equivalent to the energy input $a_{o} \gamma_{\ell g}[-\chi+(1-\chi)]$ or

$$
\Gamma_{o}=+a_{o} \gamma_{\ell g}(1-2 \chi) \text {. }
$$

The fourth energy input involves interaction with $N$ adjacent cavities of distinct filling states $\sigma_{i}$ connected to the cavity in the unit cell of interest by "necks" of cross-section $a_{n_{i}}$ with $i=1, N$. As with unsaturated porous media [15], a gas-liquid interface with energy $a_{n_{i}} \gamma_{\ell g}$ is created if cavity $i$ is empty, or destroyed otherwise. Then, the energy input to the unit cell from its neighbors is

$$
\Gamma_{n}=\sum_{i=1}^{N} \sigma_{i} \gamma_{\ell g} a_{n_{i}} .
$$

The analysis is simplified by replacing the state $\sigma_{i}$ of the many adjacent cavities by the mean field $\bar{\sigma}$, such that $\Gamma_{n}=\bar{\sigma} \gamma_{\ell g} a_{n}$, where $a_{n} \equiv \sum a_{n_{i}}$ summed over all $N$ adjacent cavities $i$. With $\sigma= \pm 1$, such mean field arises when full cavities occur at a probability $S$, and empty ones at $(1-S)$. The quantity $S=(1-\bar{\sigma}) / 2$ can therefore be interpreted either as a filling probability, or as the mean cavity saturation in liquid.

Overall, the energy change is

$$
\Delta E(\sigma=+1 \rightarrow-1)=W+\Gamma_{p}+\Gamma_{o}+\Gamma_{n} .
$$

Equations (2)-(6) suggest that energy can be made dimensionless with the mean cavity opening area $\bar{a}_{0}$ and gas-liquid surface energy $\gamma_{\ell g}, E^{*} \equiv E /\left(\bar{a}_{0} \gamma_{\ell g}\right)$.

A similar reasoning yields an equal and opposite energy input needed to empty out the cavity, $\Delta E^{*}(\sigma=+1 \rightarrow-1)=$ $-\Delta E^{*}(\sigma=-1 \rightarrow+1)$. Equivalently,

$$
\Delta E^{*}(-\sigma \rightarrow+\sigma)=\sigma\left(2 \chi-1+\alpha \cos \theta_{e}-\lambda \bar{\sigma}-\kappa^{*}\right),
$$

where

$$
\begin{gathered}
\kappa^{*} \equiv \kappa v_{p} / \bar{a}_{0}, \\
\alpha \equiv a_{p} / \bar{a}_{0},
\end{gathered}
$$

and

$$
\lambda \equiv a_{n} / \bar{a}_{0}
$$

In short, with the Ising model, the geometry of an individual cavity near the contact line is characterized by two parameters $\alpha$ and $\lambda$ representing, respectively, the specific cavity wetted area and neck cross-section. The state of a large ensemble of such cavities is defined by their probability $\chi$ of having been overcome by the contact line and by the mean filling variable $\bar{\sigma}$.

The last parameter arising in the energy budget of Eq. (8) is the aspect ratio $\kappa^{*}$. Unless cavities have size on the order of the drop scale, it is negligibly small in most cases. For example, a spherical cap sessile drop of interface radius $R$ on a horizontal surface with cylindrical cavities of depth $h$ has $\kappa^{*}=2 h / R \ll 1$. (However, if any other source of wetting fluid pressure exists, such as during the impact of a drop on a plane, the effective instantaneous value of $\kappa^{*}$ could be more significant.)

With the inconsequential choice of the ground energy state at $\bar{\sigma}=0$, cavity energy is derived from Eq. (8),

$$
E^{*}=\frac{\sigma}{2}\left(2 \chi-1+\alpha \cos \theta_{e}-\lambda \bar{\sigma}-\kappa^{*}\right) .
$$

We follow the same statistical mechanics framework outlined by $\mathrm{Xu}$ and Louge [15]. Energy and population constraints lead to a Maxwell-Boltzmann probability distribution of $\sigma$,

$$
\operatorname{Pr}(\sigma)=\frac{1}{Z} e^{-\beta^{*} E^{*}(\sigma)},
$$


where $\beta^{*} \equiv \bar{a}_{0} \gamma_{\ell g} \beta$ is a dimensionless Lagrange multiplier and $Z=\exp \left(-\beta^{*} E^{*}\right)+\exp \left(+\beta^{*} E^{*}\right)$ is the partition function. Therefore, the expected value of the filling state is

$$
\begin{aligned}
\langle\sigma\rangle & =\sum_{\sigma= \pm 1} \sigma \operatorname{Pr}(\sigma) \\
& =-\tanh \left[\frac{\beta^{*}}{2}\left(2 \chi-1+\alpha \cos \theta_{e}-\lambda \bar{\sigma}-\kappa^{*}\right)\right] \\
& =-\tanh \left[\beta^{*}\left(\chi-\chi_{c}\right)\right],
\end{aligned}
$$

where

$$
2 \chi_{c} \equiv 1-\alpha \cos \theta_{e}+\lambda \bar{\sigma}+\kappa^{*} .
$$

For unsaturated porous media, $\mathrm{Xu}$ and Louge [15] used a balance of fluid entropy to show that $\beta^{*}$ is very large $\sim 10^{11}$ for wetting fluids and porous solids of practical interest, suggesting that $\beta \sim 1 / k T$, where $k \simeq 1.3810^{-23} \mathrm{~J} /$ mole.$^{\circ} \mathrm{K}$ is Boltzmann's constant and $T$ is the absolute temperature. Here, this estimate also implies that $\beta^{*} \gg 1$. For example, even for tiny cylindrical cavities of $10 \mathrm{~nm}$ exposed to an air-water drop with $\gamma_{\ell g} \simeq 0.072 \mathrm{~J} / \mathrm{m}^{2}$ at $T=300^{\circ} \mathrm{K}, \beta^{*} \sim$ $10^{3}$ is $\gg 1$, and it grows larger with greater cavity size. In short, the ensemble of cavities experiences "frozen" disorder, equivalent to a vanishing temperature. With $\beta^{*} \gg 1$, the expected filling state of an individual cavity in Eq. (14) can then be approximated as

$$
\langle\sigma\rangle \simeq-\operatorname{sign}\left(\chi-\chi_{c}\right),
$$

which is always \pm 1 and changes sign abruptly when $\chi=$ $\chi_{c}$. Therefore, with $\beta^{*} \gg 1$, the frozen disorder arises from geometrical variations in $\lambda$ and $\alpha$ for individual cavities.

In general, there is a normalized joint statistical distribution $F(\lambda, \alpha)$ of the quantities in Eqs. (10) and (11), such that

$$
d a=A F d \lambda d \alpha
$$

is the elementary area within the total cavity opening $A$ that has $\lambda \in[\lambda, \lambda+d \lambda]$ and $\alpha \in[\alpha, \alpha+d \alpha]$. With this definition, the ensemble-average expected filling state is

$$
\overline{\langle\sigma\rangle}=\int_{\lambda=0}^{\infty} \int_{\alpha=0}^{\infty}\langle\sigma\rangle F d \lambda d \alpha=\iint \operatorname{sign}\left(\chi_{c}-\chi\right) F d \lambda d \alpha,
$$

where the overbar denotes the ensemble-average on $F$. The sign function in the double integral of Eq. (18) implies that

$$
\overline{\langle\sigma\rangle}=\mathbb{I}\left(\bar{\sigma}, \chi ; \cos \theta_{e} ; \kappa^{*}\right) \equiv \iint_{\Omega_{+}} F d \lambda d \alpha-\iint_{\Omega_{-}} F d \lambda d \alpha,
$$

where $\Omega_{+}$and $\Omega_{-}$are complementary nonintersecting regions filling the parameter space $(\lambda, \alpha)$ and having values of $(\lambda, \alpha)$ satisfying

$$
\Omega_{ \pm} \Leftrightarrow\left(\chi_{c}-\chi\right) \gtrless 0 .
$$

At a given probability $\chi$ for cavities to reside below the drop [as sketched in Fig. 1(a)], the domain $\Omega_{+}$can be interpreted as the region in the $(\lambda, \alpha)$ parameter space where cavities have not yet been filled, since they each possess a critical $\chi_{c}>\chi$. Conversely, $\Omega_{-}$is for filled cavities (Fig. 2).

For an ergodic system at equilibrium,

$$
\bar{\sigma}=\overline{\langle\sigma\rangle},
$$

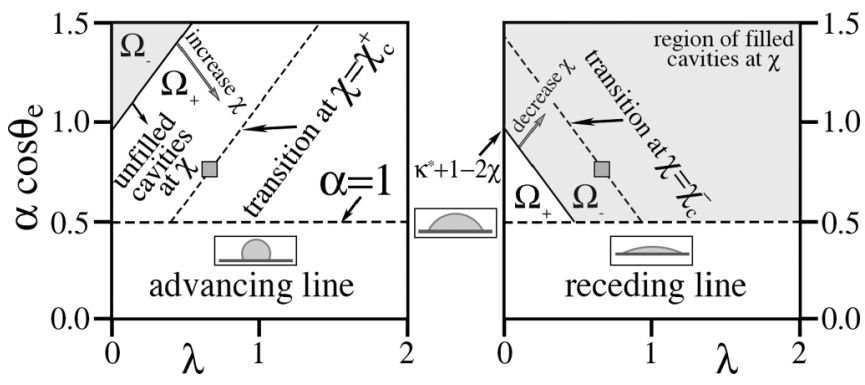

FIG. 2. $\left(\lambda, \alpha \cos \theta_{e}\right)$ parameter space plotted with $\kappa^{*}=0.05$ and $\theta_{e}=60^{\circ}$. The filled square marks an ensemble of cavities with singlevalued $\lambda$ and $\alpha$. Boundaries between the $\Omega_{-}$and $\Omega_{+}$domains are straight lines of intercept $\alpha \cos \theta_{e}=\kappa^{*}+1-2 \chi$. On the left and right figures, solid lines represent identical cavities starting with $\bar{\sigma}$ $=+1$ (dry) and $\bar{\sigma}=-1$ (wet), respectively. Increasing $\chi$ moves these boundaries downward. As they reach the $\delta$ function (filled square), a first-order phase transition occurs. At that point, cavities in the mesoscopic neighborhood have probabilities $\chi_{c}^{+}$(left figure) and $\chi_{c}^{-}$ (right) to have been overcome by the contact line. Middle inset: sketch of a drop with contact angle $\theta_{e}$. Left and right insets: sketches of $\theta_{a}$ and $\theta_{r}$ predicted by the theory in Secs. IV and IX for a bed of rods with $\epsilon=0.8$.

so that Eq. (19) can be written

$$
\bar{\sigma}=\mathbb{I}\left(\bar{\sigma}, \chi ; \cos \theta_{e} ; \kappa^{*}\right) .
$$

This implicit equation is solved to find $\bar{\sigma}$ in terms of $\chi$. When $\bar{\sigma}$ is known, we derive the expected energy of an individual cavity from Eqs. (12) and (21),

$$
\left\langle E^{*}\right\rangle=\frac{\bar{\sigma}}{2}\left(2 \chi-1+\alpha \cos \theta_{e}-\lambda \bar{\sigma}-\kappa^{*}\right) .
$$

In short, once the distribution $F(\lambda, \alpha)$ is established, the mean filling state $\bar{\sigma}$ can be calculated from Eq. (22) in terms of the Young contact angle $\operatorname{cosine} \cos \theta_{e}$, the dimensionless interface curvature $\kappa^{*}$, and the probability $\chi$ that the contact line has overcome neighboring cavities. We illustrate next how to find a solution with a simple single-valued distribution $F$.

\section{FIRST-ORDER PHASE TRANSITIONS}

As $\chi$ slowly increases or decreases, it is instructive to examine the behavior of an ensemble of cavities with identical geometry, for which $F$ is a $\delta$ function peaking at the singlevalued $(\lambda, \alpha)$ illustrated as a filled square in the parameter space of Fig. 2. In this case, because a cavity's internal area $a_{p}$ must be larger than its opening, and because $\bar{a}_{0}=a_{0}, \alpha>1$. For convenience, because $\alpha$ is always multiplied by $\cos \theta_{e}$ [for example in Eq. (12)], Fig. 2 plots this parameter space as $\left(\lambda, \alpha \cos \theta_{e}\right)$.

We begin by examining the case where all cavities are initially dry with $\bar{\sigma}=+1$ and $\chi=0$, as the contact line gradually advances over them (Fig. 2 left, and line labeled "advancing" in Fig. 3). Here, Eq. (22) is identically satisfied, since the $\delta$ function is located in domain $\Omega_{+}$(Fig. 2 left), so that Eq. (19) yields $\overline{\langle\sigma\rangle}=+1$. This dry state persists until the lower boundary of $\Omega_{+}$intersects the $\delta$ function where $\chi=\chi_{c}^{+}$ (dashed line). In this case, where $\lambda$ and $\alpha$ are single-valued,

$$
\chi_{c}^{+}=(1 / 2)\left(1-\alpha \cos \theta_{e}+\lambda+\kappa^{*}\right) .
$$




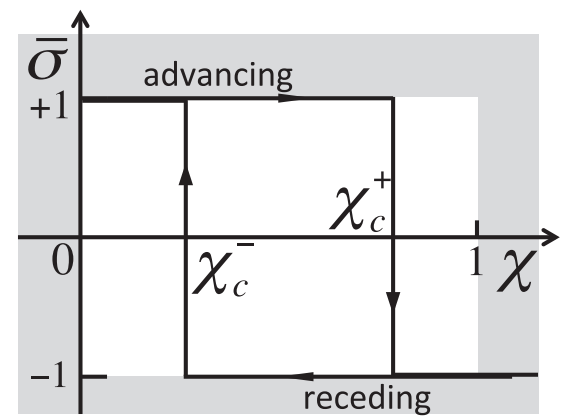

FIG. 3. Classical prediction from an Ising model without disorder: the mean filling state $\bar{\sigma}$ in the neighborhood of the contact line is plotted vs probability $\chi$ that the latter has overcome any one of its cavities, calculated for the single-valued distribution in Fig. 2 with a line advancing on initially dry cavities (left to right) or another receding on initially filled cavities (right to left). Here, the abrupt character of the two phase transitions is due to the Ising assumption that an individual unit cell can only have $\sigma= \pm 1$.

Then, for $\chi>\chi_{c}^{+}$, the $\delta$ function resides in $\Omega_{-}$, so Eq. (19) yields $\overline{\langle\sigma\rangle}=-1$. Therefore, the ensemble undergoes a phase transition at $\chi=\chi_{c}^{+}$, beyond which its state abruptly switches to $\bar{\sigma}=-1$, as all cavities in the neighborhood of the contact line fill up simultaneously.

Conversely, when all cavities are initially wet $(\bar{\sigma}=-1)$ and $\chi$ decreases from one (Fig. 3, line labeled "receding"), the ensemble satisfies Eq. (22) until it undergoes a different phase transition at $\chi=\chi_{c}^{-}$, where

$$
\chi_{c}^{-}=(1 / 2)\left(1-\alpha \cos \theta_{e}-\lambda+\kappa^{*}\right) .
$$

From Eqs. (24) and (25), we find that $\chi_{c}^{+}-\chi_{c}^{-}=\lambda$ when $\lambda$ and $\alpha$ are single-valued.

To sum up, phase transitions brings all identical cavities from $\bar{\sigma}_{i}=\varpi$ to $\bar{\sigma}_{f}=-\varpi$ at a critical probability,

$$
2 \chi_{c_{\varpi}}=1-\alpha \cos \theta_{e}+\varpi \lambda+\kappa^{*},
$$

where $\varpi \equiv+1$ and -1 for advancing and receding lines, respectively. Because these transitions involve a change in energy calculated from Eqs. (21), (23), and (26),

$$
\Delta\left\langle E^{*}\right\rangle \equiv \Delta \bar{E}^{*}=\bar{E}^{*}\left(\bar{\sigma}_{f}=-\varpi\right)-\bar{E}^{*}\left(\bar{\sigma}_{i}=+\varpi\right)=-\lambda,
$$

they are classified as first-order.

If a transition occurs, its $\Delta E^{*}$ is always exothermic, i.e., cavities give up a positive energy $-\Delta \bar{E}^{*}>0$ as the contact line either advances or recedes. However, such transition can only take place if its critical probability $\chi_{c_{\sigma}}$, which derives from $\alpha \cos \theta_{e}, \lambda$ and $\kappa^{*}$, belongs to the interval [0,1]. If instead $\chi_{c_{\sigma}} \notin[0,1]$, then the transition does not take place, and its latent energy is not released to the contact region.

Therefore, because there is a total of six possible placements of $\chi_{c}^{-}$and $\chi_{c}^{+}$relative to 0 and 1 , our theory predicts six distinct behaviors of the contact line. In the following sections, we will demarcate the corresponding six regimes in the $\left(\lambda, \alpha \cos \theta_{e}\right)$ parameter space and derive the resulting values of their distinct advancing and receding contact angles for a simple geometry. To that end, we calculate next the balance of energy given to the mesoscopic neighborhood of the contact line.

\section{POTENTIAL ENERGY}

In this section, we consider the potential energy that is supplied to a liquid spherical cap of constant volume as its contact line advances or recedes over unit cells sketched in Fig. 1. Tadmor [17] showed that, as the contact line proceeds across an increment $d A_{k \ell}$ of interfacial area between the liquid of index $\ell$ and an underlying substrate $k$, a potential energy $d G$ is given to the drop,

$$
d G=\gamma_{k \ell} d A_{k \ell}+\gamma_{\ell g} d A_{\ell g}+\gamma_{g k} d A_{g k},
$$

where interface area increments are denoted by indices of their respective phases, and $g$ denotes the gas.

In general, a change in the area between drop liquid and substrate is equal and opposite to the change in area between the gas above the drop and the same substrate, $d A_{g k}=-d A_{k \ell}$. In addition, for a spherical cap of contant volume [17], $d A_{\ell g} / d A_{k \ell}=\cos \theta$. This expression also applies to a liquid drop deformed by gravity [18]. Substituting into Eq. (28),

$$
d G=\left(\gamma_{k \ell}+\gamma_{\ell g} \cos \theta-\gamma_{g k}\right) d A_{k \ell} .
$$

For an individual unit cell (Fig. 1), the material represented by $k$ can either be solid $(k=s)$ when the contact line rides over the surface of area $a_{s}$, gas when the line sweeps across an empty cavity of area $a_{0}(k=g)$, or liquid when the latter is filled $(k=\ell)$.

As the contact line travels across the unit cell, the required energy input $\delta G=\delta G_{1}+\delta G_{2}$ has two contributions, namely from the solid surface $a_{s}$, and from the cavity opening $a_{0}$. To calculate the first, Eq. (29) is integrated over $a_{s}$ to yield $\delta G_{1}=\varpi a_{s} \gamma_{\ell g}\left(\cos \theta-\cos \theta_{e}\right)$. In this expression, $\varpi=+1$ and -1 for advancing and receding lines, which respectively increase and decrease the wetted area, thereby having equal and opposite contributions to $\delta G_{1}$.

The contribution of $a_{0}$ depends on whether the cavity is full or empty. If it is full, Eq. (29) is integrated over $a_{0}$ to yield $\varpi a_{0}\left(\gamma_{\ell \ell}+\gamma_{\ell g} \cos \theta-\gamma_{g \ell}\right)=\varpi a_{0} \gamma_{\ell g}(\cos \theta-1)$. This occurs at a probability $S=(1-\bar{\sigma}) / 2$. If it is empty, the integration yields instead $\varpi a_{0} \gamma_{\ell g}(\cos \theta+1)$. This occurs at a probability $1-S=(1+\bar{\sigma}) / 2$. Overall, the contribution of the cavity opening to the input energy is the weighted average $\delta G_{2}=\varpi a_{0} \gamma_{\ell g}[(\cos \theta+1)(1+\bar{\sigma}) / 2+$ $(\cos \theta-1)(1-\bar{\sigma}) / 2]=\varpi a_{0} \gamma_{\ell g}(\bar{\sigma}+\cos \theta)$.

We define the dimensionless solid surface area $\zeta \equiv a_{s} / \bar{a}_{0}$ and opening area $\eta \equiv a_{0} / \bar{a}_{0}$ relative to the mean cavity opening $\bar{a}_{0}$. Then, for a cavity with expected filling state $\bar{\sigma}$, the following potential energy must be supplied to overcome the unit cell $(\varpi=+1)$, or to recede from it $(\varpi=-1)$ :

$$
\varpi \delta G=\bar{a}_{0} \gamma_{\ell g}\left[\zeta\left(\cos \theta-\cos \theta_{e}\right)+\eta(\bar{\sigma}+\cos \theta)\right] .
$$

We introduce the normalized distribution $f_{\zeta}$ such that the incremental probability to find a unit cell with relative surface area in the range $[\zeta, \zeta+d \zeta]$ is $f_{\zeta} d \zeta . f_{\eta}$ is a similar increment for $\eta \in[\eta, \eta+d \eta]$. These definitions let us calculate the energy needed by the drop to overcome a unit cell at an incremental probability in the range $[\chi, \chi+d \chi]$,

$$
\begin{aligned}
\varpi d(\delta G)= & \bar{a}_{0} \gamma_{\ell g}\left[\zeta f_{\zeta}\left(\cos \theta-\cos \theta_{e}\right) d \zeta\right. \\
& \left.+\eta f_{\eta}(\bar{\sigma}+\cos \theta) d \eta\right] d \chi .
\end{aligned}
$$


We integrate this expression to find the mean potential energy supplied to the drop upon line motion over a average unit cell,

$$
\begin{aligned}
\varpi \delta \bar{G} & =\int_{\zeta} \int_{\eta} \int_{\chi} \varpi d(\delta G) \\
& =\bar{a}_{0} \gamma_{\ell g}\left[\bar{\zeta}\left(\cos \theta-\cos \theta_{e}\right)+\bar{\eta}\left(\cos \theta+\int_{\chi=0}^{1} \bar{\sigma} d \chi\right)\right],
\end{aligned}
$$

where $\bar{\eta} \equiv 1, \bar{\zeta}=\bar{a}_{s} / \bar{a}_{0} \equiv(1-\epsilon) / \epsilon$, and $\epsilon$ is the overall fraction of the flat plate surface that is made up of cavity openings. Therefore, as $\chi$ varies continuously from 0 to 1 for an advancing contact line, and conversely from 1 to 0 for a receding one, the energy $\delta \bar{G}$ supplied to the drop by an average unit cell is

$$
\varpi \epsilon \frac{\delta \bar{G}}{\bar{a}_{0} \gamma_{\ell g}}=\left[\cos \theta-(1-\epsilon) \cos \theta_{e}+\epsilon \int_{\chi=0}^{1} \bar{\sigma} d \chi\right] .
$$

To this input, we must add the latent energy $-\Delta \bar{E}$ that cavities suddenly release as they undergo a first-order phase transition at fixed $\chi=\chi_{c}$, but only if this transition occurs, i.e., if $\chi_{c} \in[0,1]$. By analogy with Haines jumps in unsaturated porous media, which dissipate a similar latent energy through viscous losses [15], the release is too fast for the underlying solid to absorb it. Therefore, this energy is entirely transferred to the drop. This contribution from collective phase transitions in the ensemble of cavities was not considered earlier [17]. Combining Eqs. (21) and (23), and integrating over the distribution $F$, the latent energy is given by

$$
\begin{aligned}
\frac{\Delta \bar{E}}{\bar{a}_{0} \gamma_{\ell g}}= & \frac{1}{2}\left(2 \chi_{c}-1+\bar{\alpha} \cos \theta_{e}-\kappa^{*}\right)\left(\bar{\sigma}_{f}-\bar{\sigma}_{i}\right) \\
& -\frac{\bar{\lambda}}{2}\left(\bar{\sigma}_{f}^{2}-\bar{\sigma}_{i}^{2}\right),
\end{aligned}
$$

if $\chi_{c} \in[0,1]$ and $\Delta \bar{E}=0$ otherwise. In Eq. (34), $\bar{\sigma}_{i}$ and $\bar{\sigma}_{f}$ are, respectively, the filling states just before and just after the transition. For an advancing line, $\sigma_{i} \equiv \bar{\sigma}^{+}$and $\sigma_{f} \gtrsim-1$; for a receding one, $\sigma_{i} \equiv \bar{\sigma}^{-}$and $\sigma_{f} \lesssim+1$. In the example of Sec. III, $\sigma_{i}= \pm 1$ and $\sigma_{f}=\mp 1$ for advancing and receding lines, respectively. As expected, Eq. (34) is compatible with Eq. (27) when $\lambda$ and $\alpha$ are single-valued.

Overall, the drop acquires acquires a potential energy $\delta \bar{G}-\Delta \bar{E}$ as it passes over an average unit cell. Dividing by $\varpi\left(\bar{a}_{0}+\bar{a}_{s}\right)$, which accounts for unit cell area as well as direction of motion, and invoking Eqs. (33) and (34), the potential energy supplied to an elementary area $d A_{s \ell}$ of a rough solid of uniform texture is

$$
d \bar{G}=d A_{s \ell} \gamma_{\ell g}\left(\cos \theta-\cos \theta_{\varpi}\right),
$$

where

$$
\cos \theta_{a} \equiv(1-\epsilon) \cos \theta_{e}-\epsilon \int_{0}^{1} \bar{\sigma} d \chi+\epsilon \Delta \bar{E}^{*}
$$

for an advancing contact line $(\varpi=+1$, index $a)$, and

$$
\cos \theta_{r} \equiv(1-\epsilon) \cos \theta_{e}-\epsilon \int_{0}^{1} \bar{\sigma} d \chi-\epsilon \Delta \bar{E}^{*}
$$

for a receding one $(\varpi=-1$, index $r$ ). The formulation of Eq. (35) resembles the change in Gibbs free energy $d \bar{G}$ of

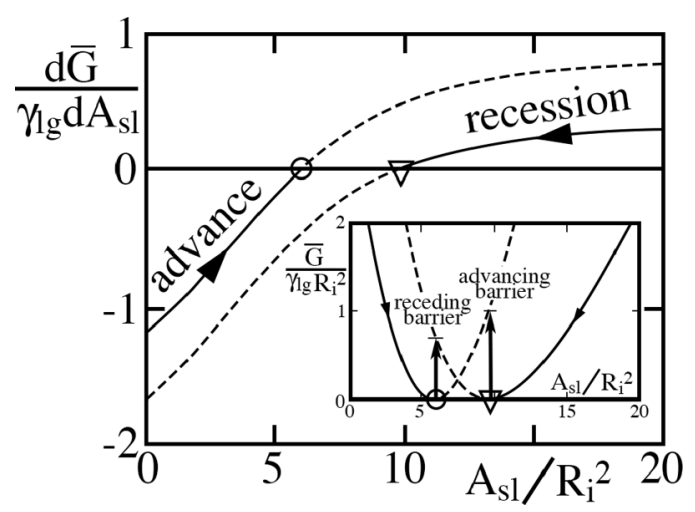

FIG. 4. Dimensionless potential energy and its derivative with respect to wetted surface area of a spherical cap. Circles and triangles illustrate, respectively, advancing and receding equilibria with $\theta_{a}=$ $4 \pi / 9$ and $\theta_{r}=5 \pi / 18$. Dashed lines are paths forbidden by Eq. (38) for drops without extrinsic energy. Main graph: $\left(1 / \gamma_{\ell g}\right) d \bar{G} / d A_{s \ell}$ from Eq. (35) vs $A_{s \ell} / R_{i}^{2}$ in Eq. (39). Inset: $\bar{G} / \gamma_{\ell g} R_{i}^{2}$ in Eqs. (40)-(42) vs $A_{s \ell} / R_{i}^{2}$. "Advancing" and "receding" barriers are $\bar{G}\left(\theta_{a}\right)$ and $\bar{G}\left(\theta_{r}\right)$.

a chemical mixture subject to a change in the degree of advancement $d \xi$ of a reaction. In such system, the law of mass action provides an equilibrium equation, in which the net sum of chemical potentials weighed by stoichiometric coefficients vanishes [19].

Here, $d A_{s \ell}$ plays the role of $d \xi$. The second law then mandates that the drop can only lose potential energy, if it is left to its own device,

$$
d \bar{G} \leq 0
$$

Figure 4 illustrates this process for a liquid drop in the shape of a spherical cap. Here, the contact patch area is

$$
\frac{A_{s \ell}}{R_{i}^{2}}=\frac{2^{4 / 3} \pi(1+t)}{(2+t)^{2 / 3}(1-t)^{1 / 3}},
$$

where $R_{i} \equiv(3 V / 4 \pi)^{1 / 3}$ is the radius of a liquid sphere having the same volume $V$ as the liquid spherical cap, and $t \equiv \cos \theta$. Taking $\bar{G}$ to vanish as $\theta$ reaches equilibrium, integration of Eq. (35) around $\theta_{\varpi}$ then yields a positive potential,

$$
\frac{\bar{G}}{\gamma_{\ell g}}=\int_{t^{\prime}=t_{\varpi}}^{t}\left(t^{\prime}-t_{\varpi}\right) \frac{\partial A_{s \ell}}{\partial t^{\prime}} d t^{\prime}=2^{4 / 3} \pi \frac{f_{n}}{f_{d}},
$$

where $t_{\varpi} \equiv \cos \theta_{\varpi}$,

$$
\begin{aligned}
f_{n}(t) \equiv & t_{\varpi} \times\left[\left(1+t_{\varpi}\right)(1-t)^{1 / 3}(2+t)^{2 / 3}\right. \\
& \left.-(1+t)\left(1-t_{\varpi}\right)^{1 / 3}\left(2+t_{\varpi}\right)^{2 / 3}\right] \\
& -2(1-t)^{1 / 3}(2+t)^{2 / 3}+2\left(1-t_{\varpi}\right)^{1 / 3}\left(2+t_{\varpi}\right)^{2 / 3},
\end{aligned}
$$

and

$$
f_{d}(t) \equiv(1-t)^{1 / 3}\left(1-t_{\varpi}\right)^{1 / 3}(2+t)^{2 / 3}\left(2+t_{\varpi}\right)^{2 / 3} .
$$

If a contact line advances over a rough solid without energy addition $\left(d A_{s \ell}>0\right)$, then Eqs. (35) and (38) imply that its angle cosine can only increase toward the equilibrium value $\cos \theta_{a}$ in Eq. (36) and its corresponding patch area from Eq. (39) (circles in Fig. 4). Conversely, if the line recedes 
$\left(d A_{s \ell}<0\right)$, then $\cos \theta$ must decrease until it reaches $\cos \theta_{r}$ in Eq. (37) (triangles in Fig. 4).

\section{PINNING}

The equilibrium relation

$$
\left(1 / \gamma_{\ell g}\right) d \bar{G} / d A_{s \ell}=\left(\cos \theta-\cos \theta_{\varpi}\right)=0
$$

resembles the law of mass action [19]. However, unlike reacting mixtures, two possible equilibria exist if $\cos \theta_{a}<$ $\cos \theta_{r}$. Figure 4 illustrates consequences on the hypothetical spreading of a liquid drop that maintains the shape of a spherical cap. Starting from a liquid sphere touching the plane with $\cos \theta=-1$, the drop progressively grows its contact patch until it reaches the equilibrium area $A_{s \ell}\left(t_{a}\right)$, where $t_{a} \equiv \cos \theta_{a}$. If it musters no other extrinsic energy (e.g., kinetic, electric, etc.), then Eq. (38) prohibits further advance, and the line remains pinned with $\theta=\theta_{a}$. To overcome pinning, the advancing drop must pack enough extrinsic energy to reach the barrier $\bar{G}\left(t_{r}\right)$ for the onset of recession, where $t_{r} \equiv \cos \theta_{r}$, as shown in the inset of Fig. 4.

Conversely, a spherical cap receding from a contact angle $\theta<\theta_{r}$ pins at $\theta_{r}$, unless its extrinsic energy is $>\bar{G}\left(t_{a}\right)$. In practical situations, a drop unable to rise above either barrier remains pinned, deforms, and dissipates excess extrinsic energy through viscous losses [20,21].

An analysis of Eqs. (40)-(42) shows that, for small differences $\Delta t \equiv t_{r}-t_{a}$ between the receding and advancing contact angle cosines, the mean energy barrier $\tilde{G} \equiv\left[\bar{G}\left(t_{a}\right)+\right.$ $\left.\bar{G}\left(t_{r}\right)\right] / 2$ grows with $\Delta t$ as

$$
\begin{aligned}
\tilde{G} / \gamma_{\ell g} R_{i}^{2}= & 2^{1 / 3} \pi \times\left[2(1-\bar{t})^{2 / 3}(2+\bar{t})^{1 / 3}\right. \\
& -(1+\Delta t-\bar{t})^{2 / 3}(2-\Delta t+\bar{t})^{1 / 3} \\
& \left.-(1-\Delta t-\bar{t})^{2 / 3}(2+\Delta t+\bar{t})^{1 / 3}\right],
\end{aligned}
$$

where $\bar{t} \equiv\left(t_{a}+t_{r}\right) / 2$ is the mean contact angle cosine. Because a line without contact angle hysteresis has no such barrier, it does not pin. Near $\Delta t \sim 0$, the barrier rises as $(\Delta t)^{2} /\left[(1-\bar{t})^{4 / 3}(2+\bar{t})^{5 / 3}\right]$. It is minimum for $\bar{t}=-1 / 3$, i.e., for a contact angle $\simeq 109.5^{\circ}$.

\section{GEOMETRICAL DISORDER}

In general, cavity geometry is random. In this case, the relation between mean filling state $\bar{\sigma}$ and probability $\chi$ must be calculated from Eqs. (19)-(22) with a broad distribution $F(\lambda, \alpha)$. Figures 5-7 illustrates this calculation with a generic $F$ sketched in Fig. 5.

Consider first a contact line advancing on a surface initially with $\bar{\sigma}=+1$ (dry) and $\chi=0$ (no cavities have yet been overcome). This state is marked by a red triangle on the upper right corner of Fig. 6 at the intersection of the diagonal [Eq. (21)] and the dashed sigmoidal curve $\mathbb{I}\left(\bar{\sigma}, 0 ; \cos \theta_{e} ; \kappa^{*}\right)$ marked $\chi=0$. As the contact line progresses, $\chi$ increases, moving dashed II curves to the right. As magnified in the upper right inset, solutions of Eq. (22) lie at the intersection of these curves with the diagonal representing the ergodic assumption Eq. (21), thereby progressively lowering $\bar{\sigma}$. With a simple causality argument [15], one can show that these solutions are stable. However, when the blue curve with $\bar{\sigma}=\bar{\sigma}^{+}$just

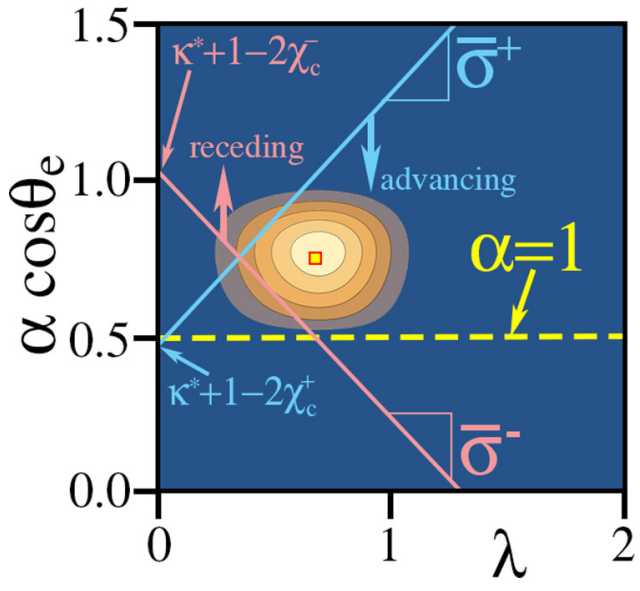

FIG. 5. Diagram of $\left(\lambda, \alpha \cos \theta_{e}\right)$ illustrated for $\kappa^{*}=0.05$, $\theta_{e}=60^{\circ}$, and an artificial normalized distribution of the form $F(\lambda, \alpha)=F_{\lambda} \times F_{\alpha}$, where $F_{\lambda} \propto\left(\lambda-d_{\lambda}\right)^{a_{\lambda} c_{\lambda}-1}$ $\exp \left\{-\left[\left(\lambda-d_{\lambda}\right) / b_{\lambda}\right]^{c_{\lambda}}\right\}$ for $\lambda>d_{\lambda}$ and zero otherwise, and $F_{\alpha} \propto\left(\alpha-d_{\alpha}\right)^{a_{\alpha} c_{\alpha}-1} \exp \left\{-\left[\left(\alpha-d_{\alpha}\right) / b_{\alpha}\right]^{c_{\alpha}}\right\}$ for $\alpha>d_{\alpha}$ and zero otherwise. With $a_{\alpha}=0.35, b_{\alpha}=0.8, c_{\alpha}=5, d_{\alpha}=1, a_{\lambda}=0.8$, $b_{\lambda}=0.8, c_{\lambda}=4$, and $d_{\lambda}=0$, the yellow square marks the mean values $(\bar{\lambda}, \bar{\alpha}) \simeq(0.669,1.51)$. The blue and red lines, with slopes and intercepts shown, mark boundaries between regions $\Omega^{-}$(above the lines) and $\Omega^{+}$(below them) for, respectively, the advancing phase transition at $\left(\chi_{c}^{+}, \bar{\sigma}^{+}\right)$and its receding counterpart at $\left(\chi_{c}^{-}, \bar{\sigma}^{-}\right)$. The horizontal dashed line shows $\alpha=1$.

tangents the diagonal (blue open circle), the system must jump to the other intersection at a lower $\bar{\sigma}$ very close to -1 , hence undergoing a collective phase transition.

Conversely, if the system starts with all cavities full of liquid $(\bar{\sigma}=-1)$ and a contact line that has overcome them all $(\chi=1)$, it remains at that filling state until $\chi$ is low, with a solution progressively rising on the diagonal from the blue disk on the lower left corner of Fig. 6, as dashed $\mathbb{I}$ curves move to the left. When $\chi$ reaches $\chi^{-}$, the red $\mathbb{I}$ curve tangents the diagonal at the red open triangle, such that any lower value of $\chi$ can no longer be a solution. At that point, the

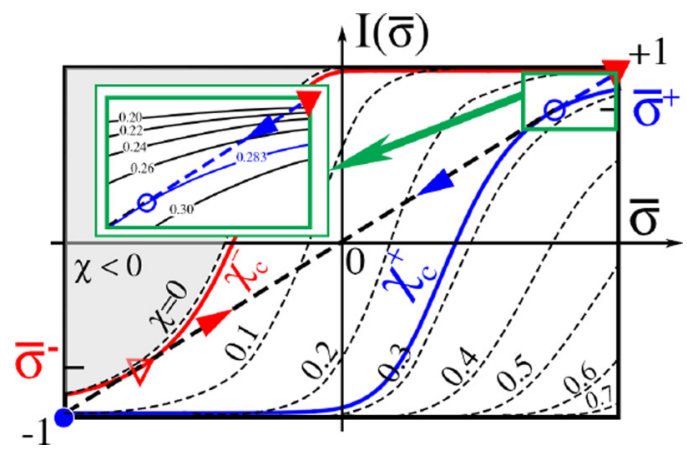

FIG. 6. Integral II from Eq. (19) vs $\bar{\sigma}$ illustrating the solution to the self-consistent Eq. (22) for the broad distribution $F$ in Fig. 5. The dashed lines are $\mathbb{I}$ at the values of $\chi$ shown. The diagonal dashed line represents the ergodic assumption Eq. (21), which must intersect the sigmoidal curves $\mathbb{I}$ for a solution. No transition can take place within the gray region $\chi<0$. 


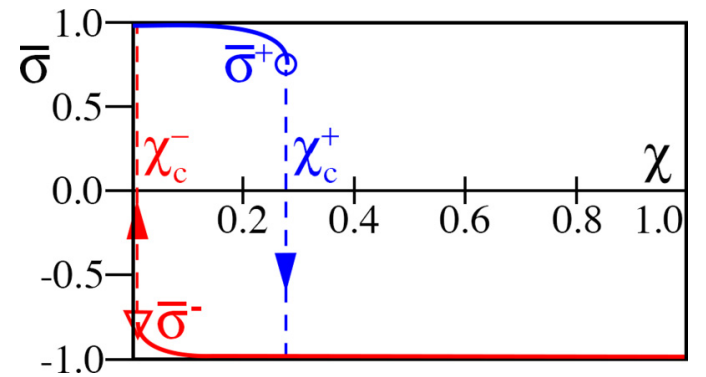

FIG. 7. Mean filling state $\bar{\sigma}$ vs probability $\chi$ for the distribution $F$ in Fig. 5. Here, the graphical solution of Fig. 6 yields $\bar{\sigma}^{+} \simeq+0.782$, $\chi_{c}^{+} \simeq 0.283, \int_{0}^{1} \bar{\sigma} d \chi \simeq-0.441$, and $\Delta \bar{E}^{*+} \simeq-0.371$ for the advancing contact line, and $\bar{\sigma}^{-} \simeq-0.778, \chi_{c}^{-} \simeq 0.013, \int_{0}^{1} \bar{\sigma} d \chi \simeq$ -0.967 , and $\Delta \bar{E}^{*-} \simeq-0.372$ for the receding one.

system undergoes another collective phase transition toward a nearly completely filled state of cavities.

As $\mathrm{Xu}$ and Louge [15] showed, the difference $\left(\chi_{c}^{+}-\chi_{c}^{-}\right)>0$ always decreases as $F$ broadens, to the point that it may disappear altogether, thereby letting a drop slide without pinning. The diagram of $\bar{\sigma}$ vs $\chi$ built from the distribution in Fig. 5 is shown in Fig. 7. Because both $\chi_{c}^{+}$and $\chi_{c}^{-} \in[0,1]$ in this case, line advance and recession experience first-order phase transitions.

In Fig. 8, we show a counter-example where $\chi_{c}^{-} \in[0,1]$ but $\chi_{c}^{+} \notin[0,1]$. In that case, the contact line advancing on a dry rough surface can only induce a partial filling of cavities to $\bar{\sigma}_{f}$ without ever releasing latent energy. The resulting absence

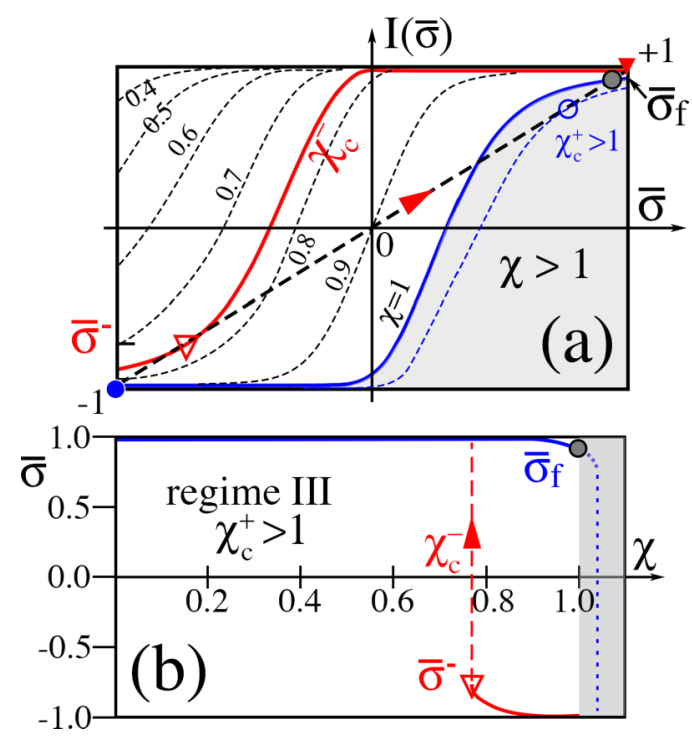

FIG. 8. The same distribution $F(\lambda, \alpha)$ as in Fig. 5 but with $\theta_{e}=$ $120^{\circ}$. In this case, the advancing transition never undergoes a phase transition $\left(\Delta \bar{E}^{*+} \equiv 0\right)$. Instead, it eventually reaches $\bar{\sigma}_{f} \simeq 0.928$ at $\chi=1$ (dark disk), where $\simeq 7.2 \%$ of cavities remain unfilled, with $\int_{0}^{1} \bar{\sigma} d \chi \simeq 0.997$. If one condensed water vapor to wet all cavities, then a receding contact line would induce a phase transition at $\chi_{c}^{-} \simeq$ 0.767 with $\bar{\sigma}^{-} \simeq-0.782, \Delta \bar{E}^{*-} \simeq-0.371, \int_{0}^{1} \bar{\sigma} d \chi \simeq 0.541$. If not, the absence of advancing transition should produce a metastable recession with greater $\theta_{r}$. (a) and (b) have the same axes as Figs. 6 and 7 , respectively. of $\epsilon \Delta \bar{E}^{*}<0$ from Eq. (36) raises $\cos \theta_{a}$, thereby changing the behavior of the contact line. We explore the diversity of regime behaviors next.

\section{REGIMES}

The existence of a latent energy implies that rough surfaces behave differently if their phase transitions take place or not. As mentioned in Sec. III, this is determined by the position of the transition probabilities $\chi_{c}^{+}$and $\chi_{c}^{-}$relative to the interval $[0,1]$. Whereas Figs. 5-7 illustrate a situation in which $F(\lambda, \alpha)$ and $\cos \theta_{e}$ lead to $\chi_{c}^{-}$and $\chi_{c}^{+} \in[0,1]$, Fig. 8 shows another case when $\chi_{c}^{+}$does not. In general, surface behavior is associated with one of six regions in the $\left(\lambda, \alpha \cos \theta_{e}\right)$ parameter space, corresponding to the six possible placements of $\chi_{c}^{-}<\chi_{c}^{+}$in $[0,1]$.

To illustrate these six distinct regimes, we now return to the simpler case of single-valued $(\lambda, \alpha)$ introduced in Sec. III. Tables I and II summarize predictions. For each regime, Table I lists the initial and final filling states $\bar{\sigma}_{i}$ and $\bar{\sigma}_{f}$, the integral of $\bar{\sigma}$ with $\chi$ and, if a phase transition occurs, the liberated energy $-\Delta \bar{E}^{*}$. Table II presents the two equilibrium contact angle $\operatorname{cosines} \cos \theta_{a}$ and $\cos \theta_{r}$ as differences with the term $(1-\epsilon) \cos \theta_{e}$ that they have in common. It also specifies whether $\chi_{c}^{-}$and $\chi_{c}^{+}$belong to the interval $[0,1]$. Because these two quantities depend on $\lambda$ and $\alpha \cos \theta_{e}$ through Eqs. (24) and (25), their positions relative to $[0,1]$ define six regions of the $\left(\lambda, \alpha \cos \theta_{e}\right)$ parameter space separated by straight lines of slopes \pm 1 . Figure 9 shows the corresponding subdivisions for the six regimes.

In regime I, both phase transitions are possible (Fig. 3). Here, the contact angle hysteresis $\left(\cos \theta_{r}-\cos \theta_{a}\right)=4 \epsilon \lambda$ explicitly depends on specific neck cross-section $\lambda$ and fractional coverage $\epsilon$ of cavities on the surface.

In regime II, the critical $\chi_{c}^{-}$is negative, so the receding phase transition is pushed to the left of Fig. 3, and therefore it cannot occur. Here, all cavities remain wet after the contact line has receded $\left(\bar{\sigma}_{f}=-1\right)$. However, the advancing transition is possible, since $\chi_{c}^{+} \in[0,1]$.

In regime III, it is instead the advancing phase transition that is forbidden $\left(\chi_{c}^{+}>1\right)$, while the receding one is allowed. As discussed in the next section, this prohibition leads to recession metastability.

In regime $\mathrm{IV}$, both advancing and receding contact lines have forbidden transitions residing at $\chi_{c}^{-}<\chi_{c}^{+}<0$, so that no latent energy is released $\left(\Delta \bar{E}^{*}=0\right)$. Here, the receding contact line travels on cavities that remain full throughout successive equilibria as $\chi$ decreases from 1 to $0\left(\bar{\sigma}=\bar{\sigma}_{i}=\right.$ $\left.\bar{\sigma}_{f}=-1\right)$. However, conversely, cavities that are initially dry are not at equilibrium in regime IV. Instead, as a contact line overcomes these cavities, they quickly fill up to reach their only possible equilibrium at $\bar{\sigma}_{i}=-1$. In other words, no equilibrium advancing contact angle can be observed with $\bar{\sigma}_{i}=+1$ in regime IV. Instead, both advance and recession equilibria possess the same initial and final filling states $\left(\bar{\sigma}_{i}=\bar{\sigma}=\bar{\sigma}_{f}=-1\right)$, and are therefore indistinguishable (no hysteresis). This regime whereby all cavities end up full of liquid is sometimes called the "wet Cassie state." Although the fractional surface coverage $\epsilon$ is the only explicit variable in 
TABLE I. Parameters in Eqs. (36) and (37) for single-valued $(\lambda, \alpha)$. If a phase transition occurs with $0 \leq \chi_{c} \leq 1, \Delta \bar{E}^{*}=-\lambda$ from Eq. (27); otherwise, $\Delta \bar{E}^{*} \equiv 0$. (a) As discussed in Sec. VIII, regimes III and V can have metastable recession, whereby values in the four rightmost columns are replaced by their advancing counterparts on the left.

\begin{tabular}{|c|c|c|c|c|c|c|c|c|}
\hline \multirow[b]{2}{*}{ Regime } & \multicolumn{4}{|c|}{ Advance $(\varpi=+1)$} & \multicolumn{4}{|c|}{ Recession $(\varpi=-1)$} \\
\hline & $\bar{\sigma}_{i}$ & $\bar{\sigma}_{f}$ & $\int_{0}^{1} \bar{\sigma} d \chi$ & $-\Delta \bar{E}^{*}$ & $\bar{\sigma}_{i}$ & $\bar{\sigma}_{f}$ & $\int_{0}^{1} \bar{\sigma} d \chi$ & $-\Delta \bar{E}^{*}$ \\
\hline I & +1 & -1 & $-\alpha \cos \theta_{e}+\lambda+\kappa^{*}$ & $\lambda$ & -1 & +1 & $-\alpha \cos \theta_{e}-\lambda+\kappa^{*}$ & $\lambda$ \\
\hline II & +1 & -1 & $-\alpha \cos \theta_{e}+\lambda+\kappa^{*}$ & $\lambda$ & -1 & -1 & -1 & 0 \\
\hline III $^{\mathrm{a}}$ & +1 & +1 & +1 & 0 & -1 & +1 & $-\alpha \cos \theta_{e}-\lambda+\kappa^{*}$ & $\lambda$ \\
\hline IV & -1 & -1 & -1 & 0 & -1 & -1 & -1 & 0 \\
\hline $\mathrm{V}^{\mathrm{a}}$ & +1 & +1 & +1 & 0 & -1 & -1 & -1 & 0 \\
\hline VI & +1 & +1 & +1 & 0 & +1 & +1 & +1 & 0 \\
\hline
\end{tabular}

$\cos \theta_{a}=\cos \theta_{r}$, values of $\lambda$ and $\alpha$ implicitly determine whether the roughness geometry belongs to this regime.

No phase transition occurs in regime V. Because $\chi_{c}^{-}$and $\chi_{c}^{+}$ reside on opposite sides of the $[0,1]$ interval, the initial filling state must remain unchanged during passage of the contact line. Like regime III, the absence of a spontaneous advancing transition can produce a metastable recession.

Finally, regime VI mirrors regime IV. Here, it is now the receding line that cannot achieve an equilibrium contact angle with full cavities. Instead, cavities at equilibrium must stay dry during both advancing or receding processes $\left(\bar{\sigma}_{i}=\bar{\sigma}=\bar{\sigma}_{f}\right.$ $=+1$ ). It is the "Cassie-Baxter" (a.k.a., "fakir Cassie") state [22] devoid of hysteresis.

\section{METASTABILITY}

Predictions in Tables I and II presume that an advancing contact line invades an initially bone-dry surface $\left(\bar{\sigma}_{i}=+1\right)$, while a receding one proceeds over fully wetted cavities $\left(\bar{\sigma}_{i}=-1\right)$, with the exception of the "Cassie" regimes IV and VI, where such initial states are not at equilibrium. However, while it is relatively easy to stage a dry surface ahead of an advancing line, it is harder to guarantee uniform cavity saturation at the onset of recession.

In traditional goniometry, receding contacts are observed after a liquid drop has previously advanced on a dry surface. This procedure poses a particular problem for regimes III and $\mathrm{V}$, in which the advance does not spontaneously transition to fully wetted cavities. Therefore, in these regimes, the equilibrium receding angles in Table II cannot be observed by withdrawing a drop that had previously invaded a dry surface. Instead, traditional goniometry detects a metastable recession that is nearly the reverse of the advancing process in regimes III or V.

In order to expose equilibrium recession in these regimes, other techniques must be used to wet all cavities beforehand. To that end, Lafuma and Quéré [23] condensed water vapor uniformly on a textured surface belonging to regime $\mathrm{V}$ until a drop formed. This led to a much smaller receding angle than observed in traditional goniometry. They obtained similar results by applying higher capillary pressure to force water into cavities, then by evaporating the drop to initiate its recession [24].

\section{BED OF RODS}

To illustrate the six regimes in Tables I and II, it is instructive to analyze a well-controlled microscopic roughness that is used to test models and produce extreme hydrophobia [23-25]. To that end, we consider an artificially textured periodic square array consisting of identical cylindrical rods of height $h^{*}$ and diameter $d^{*}$, separated by a unit distance, and rising perpendicularly from a plane of the same material. In this configuration, geometrical parameters are

$$
\begin{gathered}
\epsilon=1-(\pi / 4) d^{* 2}, \\
\lambda=16\left(1-d^{*}\right) h^{*} /\left(4-\pi d^{* 2}\right),
\end{gathered}
$$

and

$$
\alpha=1+\pi d^{*} \lambda /\left[4\left(1-d^{*}\right)\right] .
$$

Note that, because there are only two independent aspect ratios in this configuration, prescribing $(\lambda, \alpha)$ dictates $d^{*}=$ $4(\alpha-1) /[4(\alpha-1)+\pi \lambda]$ and $\epsilon$. Therefore, predictions illustrated in Fig. 9 are not universal.

TABLE II. Range of parameters in Eqs. (24) and (25) demarcating regimes I through VI, and contact angle cosines in Eqs. (36) and (37), minus the common contribution $(1-\epsilon) \cos \theta_{e}$ from the exposed solid. Remark (a), see Table I.

\begin{tabular}{lcccr}
\hline \hline Regime & $\chi_{c}^{+}$ & $\chi_{c}^{-}$ & $\cos \theta_{a}-(1-\epsilon) \cos \theta_{e}$ & $\cos \theta_{r}-(1-\epsilon) \cos \theta_{e}$ \\
\hline $\mathrm{I}$ & $\epsilon[0,1]$ & $\epsilon[0,1]$ & $+\epsilon\left(\alpha \cos \theta_{e}-2 \lambda-\kappa^{*}\right)$ & $+\epsilon\left(\alpha \cos \theta_{e}+2 \lambda-\kappa^{*}\right)$ \\
II & $\epsilon[0,1]$ & $<0$ & $+\epsilon\left(\alpha \cos \theta_{e}-2 \lambda-\kappa^{*}\right)$ & $+\epsilon$ \\
III $^{\mathrm{a}}$ & $>1$ & $\epsilon[0,1]$ & $-\epsilon$ & $+\epsilon\left(\alpha \cos \theta_{e}+2 \lambda-\kappa^{*}\right)$ \\
IV & $<0$ & $<0$ & $+\epsilon$ & $+\epsilon$ \\
$\mathrm{V}^{\mathrm{a}}$ & $>1$ & $<0$ & $-\epsilon$ & $+\epsilon$ \\
$\mathrm{VI}$ & $>1$ & $>1$ & $-\epsilon$ & $-\epsilon$ \\
\hline \hline
\end{tabular}




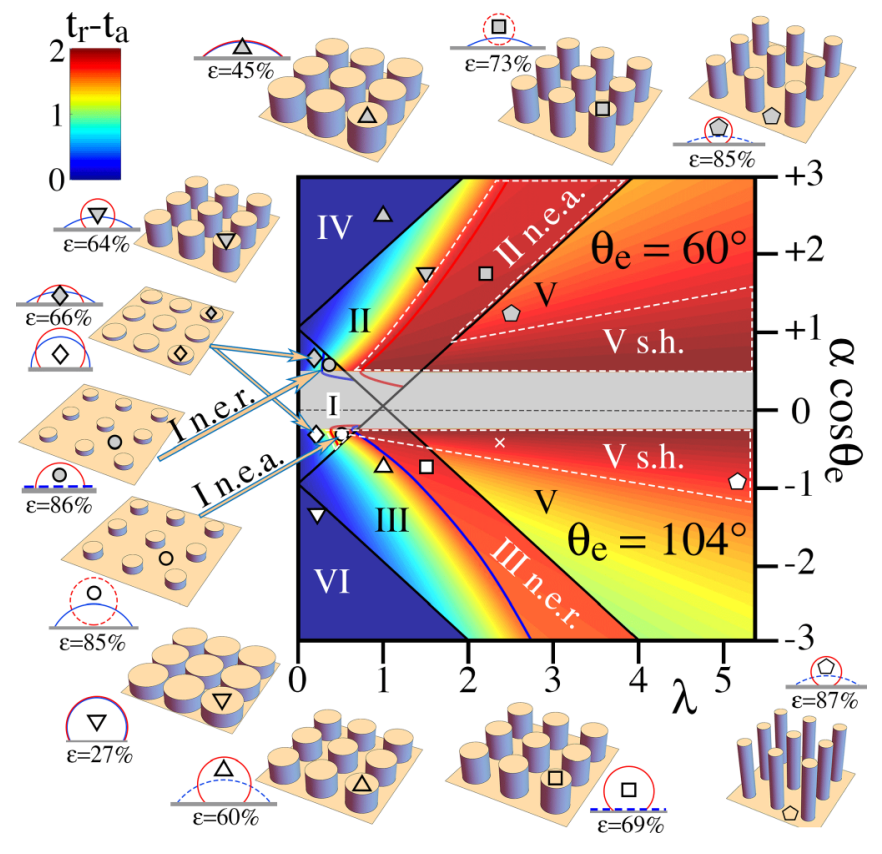

FIG. 9. Parameter space $\left(\lambda, \alpha \cos \theta_{e}\right)$ for beds of rods with singlevalued $\lambda$ and $\alpha$ in Eqs. (46) and (47), drawn for a hydrophilic solid with $\theta_{e}=60^{\circ}$ above the $\alpha=0$ axis, and for hydrophobic $\theta_{e}=104^{\circ}$ below. Black straight lines of slopes \pm 1 delimit six regimes. These lines are obtained by expressing the relative positions of $\chi_{c}^{-}$and $\chi_{c}^{+}$in Table II in terms of $\lambda$ and $\alpha \cos \theta_{e}$ using Eqs. (24) and (25). The gray region with $\alpha<1$ is geometrically impossible. Regions of nonequilibrium advancing (n.e.a.) contact are found in regimes I and II, with $\cos \theta_{a} \equiv-1$ on red lines. Nonequilibrium receding (n.e.r.) contacts also exist in regimes I and III, with $\cos \theta_{r} \equiv+1$ on blue lines. White dashed lines surround superhydrophobic (s.h.) regions where $\theta_{a}>150^{\circ}$ in regimes I, II, and V. Symbols are used to match positions in the diagram with sketches of the corresponding bed of rods and their spherical liquid caps for advancing (red) and receding (blue) contact angles found in Table II at the $\epsilon$ imposed by Eq. (45); dashed blue caps denote metastable recession or n.e.r. states; dashed red caps are n.e.a. states. The white cross is the data of Lafuma and Quéré [23,24]. The background color scale on the upper left shows hysteresis strength $t_{r}-t_{a} \equiv \cos \theta_{r}-\cos \theta_{a}$; at equilibrium, deep blue indicates no hysteresis; deep red maximum hysteresis.

A possible challenge in evaluating $\alpha$ is that meniscii forming at the opening of a cavity and at its necks may be curved, and therefore larger than the corresponding dry cross-sections. By analogy with cylindrical capillaries, $\mathrm{Xu}$ and Louge [15] showed that the ratio of meniscii area to dry cross-section is approximately $2 /\left(1+\sin \theta_{e}\right)$. Because $\bar{a}_{0}$ and $a_{n}$ are subject to the same correction, but $a_{p}$ is not, we expect that $\alpha$ alone may be affected, such that its value would effectively be smaller than $a_{p} / \bar{a}_{0}$ by a factor $\left(1+\sin \theta_{e}\right) / 2$. Another challenge to the meaning of Eqs. (46) and (47) is that cavities formed by a "bed of rods" should neither be too deep nor too widely spaced to uphold the Ising assumption of no partial filling. Nonetheless, as Fig. 9 suggests, all six regimes can be explored by changing the specific areas $\lambda$ and $\alpha$ in Eqs. (46) and (47) for solids with known Young contact angle cosine $\cos \theta_{e}$.
Beside partitioning the $\left(\lambda, \alpha \cos \theta_{e}\right)$ parameter space in six regions, Fig. 9 shows where one can expect superhydrophobia, which is typically understood as advancing angles $>150^{\circ}$ [26]. As observed for natural or artificial surface textures [26-29], a curious prediction is that superhydrophobic surfaces can be made of an inherently hydrophilic solid by exploiting textures within the top region bound by a white dashed line in Fig. 9.

The theory also finds textures that have expressions of $\cos \theta_{a}<-1$ or, equivalently, that cannot sustain an advancing angle at equilibrium. Because $-1 \leq(1-\epsilon) \cos \theta_{e} \pm 1 \leq+1$, such nonequilibrium advance does not arise in regimes III to VI. However, it is possible for areas within regimes I and II marked "I n.e.a." and "II n.e.a." (nonequilibrium advance). Similarly, Fig. 9 delimits regions of nonequilibrium recession (n.e.r.) within regimes I and III, where the expression for $\cos \theta_{r}$ rises above unity. However, because regime III has metastable recession, one is unlikely to observe perfectly wetting behavior in that regime.

Finally, the background color in Fig. 9 shows hysteresis strength calculated as the difference between the two equilibrium contact angle cosines $t_{r}-t_{a} \equiv \cos \theta_{r}-\cos \theta_{a}$.

\section{COMPARISON WITH DATA}

In this section, we invoke four data sets to test the theory. First, we make quantitative predictions for artificially textured geometries designed for superhydrophobia [23-25]. Then, we consider the hysteretic "Wenzel state" of a hydrophilic rough solid [30]. To illustrate the role of specific neck cross-section $\lambda$, we turn to the experiments of Onda, Shibuichi et al. [31,32], who recorded $\cos \theta_{a}$ versus $\cos \theta_{e}$. These data also allow us to show contact angle variations as $\alpha \cos \theta_{e}$ moves across regimes IV, II, I, III, and VI at constant $\lambda$. Finally, we show that the theory captures the behavior of superhydrophobic "reentrant" geometries $[33,34]$ having a sharp transition between regimes $\mathrm{V}$ and II.

Because our $a b$ initio theory only requires knowledge of cavity geometry and underlying Young contact angle, a bed of rods is a quantitative test of its merits. Lafuma and Quéré [23] created a sparse bed of $2 \mu \mathrm{m}$ rods with surface fraction $\epsilon \simeq$ 0.99 rising $12 \mu \mathrm{m}$ above a flat solid having a $110^{\circ}$ advancing contact angle. This geometry belongs to the superhydrophobic region of regime $\mathrm{V}$ with $\lambda \simeq 2.4$ and $\alpha \cos \theta_{e} \simeq-0.42$ (white cross in Fig. 9). Whereas they reported $\cos \theta_{a} \simeq-0.961$ for the advance, we predict $\cos \theta_{a} \simeq-0.993$. Before observing recession, these authors achieved a consistent initial wetting by condensing water vapor on the bed. This led to $\cos \theta_{r} \simeq$ +0.81 , while we calculate $\cos \theta_{r} \simeq+0.98$ from $\sigma_{i}=-1$. Our predictions have somewhat greater hysteresis strength than what they observed $\left(\cos \theta_{r}-\cos \theta_{a} \simeq 1.98\right.$ versus 1.77$)$, perhaps because the wide separation of their rods challenges our simple view of meniscii stretching across them (Fig. 1).

These data also illustrate the metastability discussed in Sec. VIII. With traditional goniometry, Lafuma and Quéré [23] recorded much larger receding angles $\left(\cos \theta_{r} \simeq-0.93\right)$ than upon condensing water vapor uniformly on the surface beforehand $\left(\cos \theta_{r} \simeq+0.81\right)$.

For their part, Gao and McCarthy [25] induced recession after depositing a drop on a similar bed of square rods 


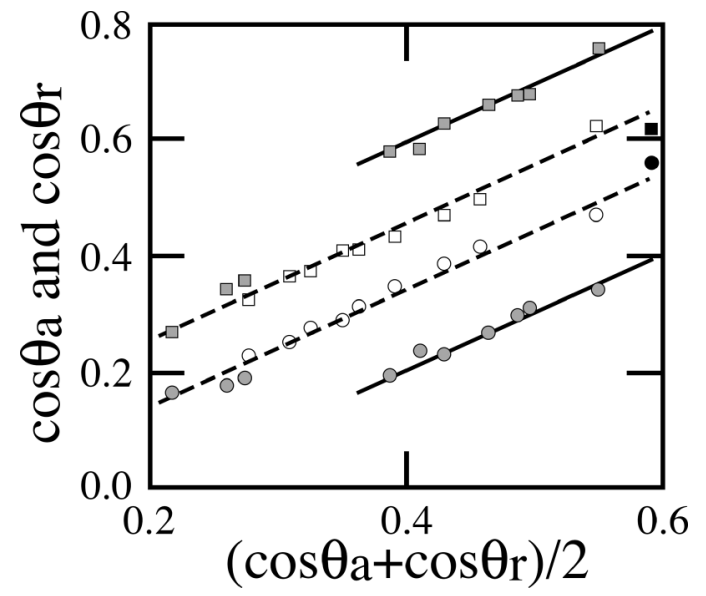

FIG. 10. Receding and advancing contact angles cosines of Lam et al. [30] vs their mean value. Circles and squares are $\cos \theta_{a}$ and $\cos \theta_{r}$. Gray-filled and open symbols, respectively, represent alcohols and alkanes as wetting liquids. The two black symbols are for octamethycyclotetrasiloxane. Solid and dashed lines are predictions in Table II with contact angle hysteresis $\cos \theta_{r}-\cos \theta_{a}=4 \bar{\lambda} \epsilon \simeq$ 0.38 and 0.11 . It is unclear why points acquired with alcohol at $\overline{\cos \theta}<0.28$ have smaller hysteresis than those with $\overline{\cos \theta}>0.38$.

belonging to the superhydrophobic region of regime $\mathrm{V}$, with $\cos \theta_{e} \simeq-0.242, \lambda \simeq 5.16$ and $\alpha \cos \theta_{e} \simeq-0.91$ (white pentagon Fig 9). For the advancing process, our prediction of $\cos \theta_{a} \simeq-0.903$ is nearly as hydrophobic as what they reported $\left(\cos \theta_{a} \simeq-0.996\right)$. However, their highly hydrophobic receding angle $\left(\cos \theta_{r} \simeq-0.83\right)$ suggests a metastable state similar to what Callies and Quéré observed before wetting cavities thoroughly $[23,24]$.

To illustrate how our predictions capture the hysteretic "Wenzel state," Fig. 10 shows the data of Lam et al. [30] for $\cos \theta_{a}$ and $\cos \theta_{r}$ on FC-732-coated silicon wafer $\langle 100\rangle$ surfaces. Because these authors did not measure $\cos \theta_{e}$ directly, we plot the dependence of the two cosines on their mean value $\overline{\cos \theta} \equiv\left(\cos \theta_{a}+\cos \theta_{r}\right) / 2$. Without a detailed geometrical characterization of the solid surface, we cannot reconstruct the distribution $F(\lambda, \alpha)$ from these experiments. However, predictions in Table II for a single-valued $F$ can yield estimates for $\lambda$ and $\alpha$. While other geometrical combinations could also match data, the results are consistent with regime $\mathrm{I}, \bar{\alpha} \simeq 1.5$, $\kappa^{*}=0$, and $\epsilon \simeq 0.5$. Experiments with alcohol and alkanes imply contact angle hysteresis of $\cos \theta_{r}-\cos \theta_{a}=4 \bar{\lambda} \epsilon \simeq$ 0.38 and 0.11 , respectively.

The "Wenzel state" derives from his 1936 theory [35], whereby the effective solid surface is augmented with the mean area $\bar{a}_{p}$ within cavities. This implies $\cos \theta=r \cos \theta_{e}$, where $r>1$ is a roughness factor. Our model approximates the factor as $r \sim(1-\epsilon)+\epsilon \alpha$ within a region of Fig. 9 roughly spanning moderate values of $\alpha$ and $\lambda$ in regimes I, II, and III.

Although the hysteresis of the "Wenzel state" grows with $r$ until it transitions to the Cassie-Baxter regime [36], the direct proportionality between contact angle cosine and $\cos \theta_{e}$ in Wenzel's theory does not explicitly distinguish between advancing and receding angles. As the background color in Fig. 9 shows, our predictions confirm that hysteresis permeates

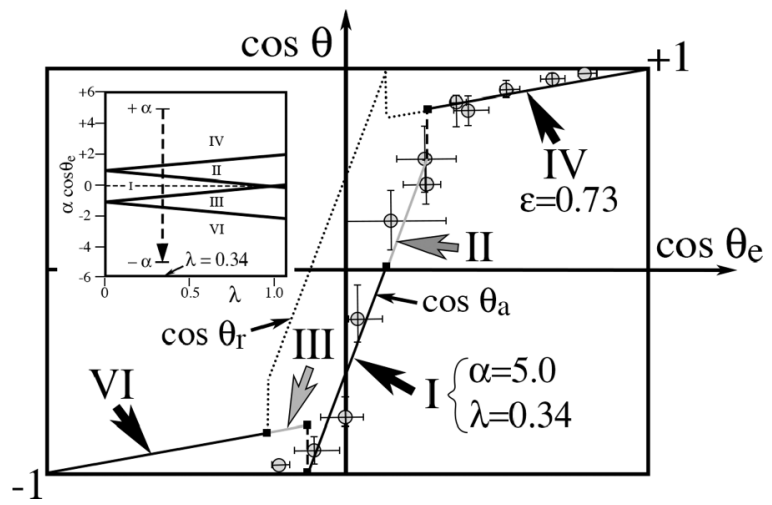

FIG. 11. Cosine of contact angles of water and 1,4 dioxane liquid mixtures on fractal alkylketene dimer surfaces vs cosine of the corresponding equilibrium angles $\cos \theta_{e}$ measured on the same flat surfaces [31,32] (symbols). We follow Quéré [9] in interpreting these contact angles as advancing. Lines are the model in Table II for the regimes shown; the solid line is $\cos \theta_{a}$ and the dotted one is $\cos \theta_{r}$. Assuming $\kappa^{*}=0$, slopes and intercepts of measured $\cos \theta_{a} \mathrm{vs} \cos \theta_{e}$ yield $\epsilon=0.73 \pm 0.09$ from points in regime IV, and $\lambda=0.34 \pm 0.15$ and $\alpha=5.0 \pm 1.8$ from regimes I and II. Over the range of $\cos \theta_{e}$ from highest to lowest (inset, dashed arrow), we predict that $\cos \theta_{a}$ successively explores regimes IV, II, I, III, and VI in the $\left(\lambda, \alpha \cos \theta_{e}\right)$ parameter space. Changes in $\cos \theta_{a}$ from a regime to the next are marked by black squares. They are smooth from II to I and III to VI, but abrupt (dashed lines) from IV to II and I to III. Data suggest a linear dependence of $\cos \theta_{a}$ that does not pass through the origin. In our model, such offset is due to $\lambda>0$.

the Wenzel state, but disappears in the "wet Cassie" and "fakir Cassie" regimes IV and VI.

Our model shows that hysteresis only arises at finite values of the specific neck cross-section $\lambda$ that produce collective interactions among neighboring cavities. For real surfaces, we do not expect $\lambda$ to be as wide as it is with an artificial bed of rods. However, as the experiments of Onda, Shibuichi et al. [31,32] demonstrated, $\lambda$ does not vanish for a rough solid. Figure 11 shows one of the three data sets that these authors obtained for a contact line advancing on solids made up of different ratios of alkylketene dimer (AKD) to dialkylketone (DAK) wax. Here, $\cos \theta_{e}$ was varied by producing different liquid mixtures of water and 1,4 dioxane. The authors managed to record this cosine on a flat AKD surface. By changing $\cos \theta_{e}$ from its highest to lowest values, their experiments successively explored regimes IV, II, I, III, and VI in the $\left(\lambda, \alpha \cos \theta_{e}\right)$ parameter space (shown in the inset of Fig. 11, downward along the dashed arrow).

As Table II indicates, $\cos \theta_{a}$ is a straight line function of $\cos \theta_{e}$ that only depends on $\epsilon$ in regime IV. Because slope and intercept of Onda, Shibuichi et al. [31,32] were both consistent with the same $\epsilon=0.73 \pm 0.09$, it is likely that their five data points at $\cos \theta_{e}>0.35$ conformed to the "wet Cassie" state that we call regime IV.

Next, the theory predicts that, as $\cos \theta_{e}$ is further reduced, the advancing angle $\operatorname{cosine} \cos \theta_{a}$ must abruptly jump as contact line behavior transitions from regime IV to regime II. Although the experiments of Onda, Shibuichi et al. [31,32] did not produce enough values of $\cos \theta_{e}$ to know with certainty 
TABLE III. Values of $\epsilon, \lambda$, and $\alpha$ estimated by matching predictions of $\cos \theta_{a}$ in Table II with three data sets [31,32], as done in Fig. 11. The first column shows AKD:DAK ratios used in experiments. Errors are $95 \%$ confidence intervals.

\begin{tabular}{lccc}
\hline \hline AKD:DAK & $\epsilon$ & $\lambda$ & $\alpha$ \\
\hline $100: 0$ & $0.726 \pm 0.085$ & $0.339 \pm 0.153$ & $5.04 \pm 1.81$ \\
$90: 10$ & $0.852 \pm 0.047$ & $0.14(+0.28,-0.14)$ & $4.5 \pm 3.1$ \\
$80: 20$ & $0.896 \pm 0.007$ & $0.10(+0.17,-0.10)$ & $3.6 \pm 1.8$ \\
\hline \hline
\end{tabular}

whether such sharp jump exists, and although a real surface with actual breadth in the distribution $F(\lambda, \alpha)$ should also weaken this jump, their data suggested that $\cos \theta_{a}$ collapses from $\sim 0.80$ down to $\sim 0.55$, with sharp break in the slope of the $\cos \theta_{a}$ versus $\cos \theta_{e}$ line. Future experiments staging a textured bed of rods and a drop subject to electrowetting $[37,38]$ would produce finer increments in $\cos \theta_{e}$ to resolve the jump.

In contrast, there is no such change in the expression of $\cos \theta_{a}$ between regimes II and I. Within experimental error, both conform to $\alpha \simeq 5.0 \pm 1.8$ and $\lambda=0.34 \pm 0.15$, which are associated, respectively, with the slope and intercept of $\cos \theta_{a}=\cos \theta_{e}[1+\epsilon(\alpha-1)]-2 \epsilon \lambda-\epsilon \kappa^{*}$. Notably, the data imply that the straight line of $\cos \theta_{a}$ versus $\cos \theta_{e}$ does not pass through the origin in regimes I and II. As Table III shows, an intercept also arises in the other two data sets of Onda, Shibuichi et al. [31,32]. Because this intercept is due to $\lambda \neq 0$, this observation suggests that the notion of surface cavities interacting through narrow necks (Fig. 1) has merit.

As expected, the theory predicts identical $\theta_{r}$ and $\theta_{a}$ for perfectly smooth surfaces. It also finds that hysteresis arises only if $\lambda \neq 0$, whether the expression for $\left(\cos \theta_{r}-\cos \theta_{a}\right)$ involves $\lambda$ or not. In addition, because the specific geometry captured by $\lambda$ and $\alpha$ does not explicitly depends on a length scale, the contact angle behavior should not depend on mean cavity size (as long as $\kappa^{*} \ll 1$ and enough fluid molecules fill a cavity). Therefore, in practice, it may be difficult to eliminate hysteresis by smoothing the solid plane alone, if invisible roughness persists on the small scale.

Last, the theory sheds light on the data of Liu and Kim [33] for a periodic "reentrant" structure designed for extreme hydrophobia. As Fig. 12 illustrates, the resulting surface roughness possessed relatively large $\lambda \simeq 2.02$ and small $\alpha \simeq$ 1.12. This produced a linear variation of $\cos \theta_{a}$ with $\cos \theta_{e}$ farther from the origin than the surfaces of Onda, Shibuichi et al. $[31,32]$ in Fig. 11 or Table III. Such $\lambda$ and $\alpha$ also induced a sharp transition of $\cos \theta_{a}$ between regimes V and II. However, because the transition occurred at a $\cos \theta_{e} \equiv(\lambda-1) / \alpha$ near 1 , subtle changes in the geometry could have suppressed it altogether. Although Liu and Kim [33] offered other reasons, such suppression may explain why their peculiar "doubly reentrant" structure remained in regime V. Finally, because regime V is metastable (Sec. VIII), Liu and Kim [33] could not readily observe its underlying equilibrium hysteresis [23]. Instead, $\cos \theta_{r}$ remained near $\cos \theta_{a}$ in a state dependent on the initial wetting $\bar{\sigma}_{i}$ that they effectively achieved at the onset of recession.

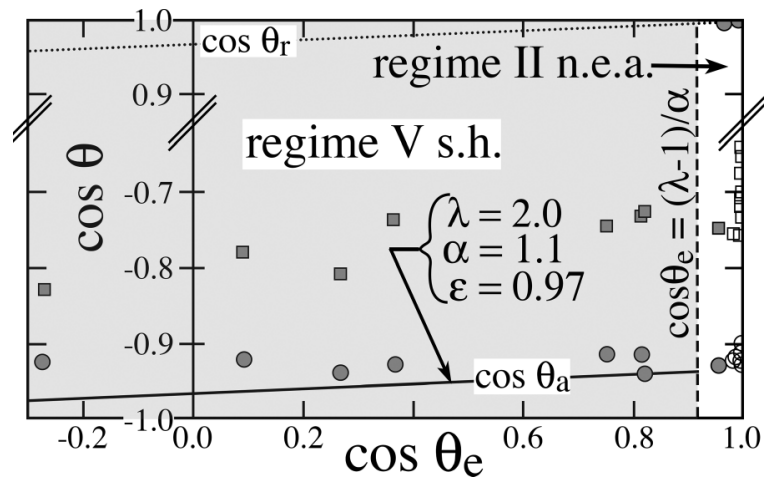

FIG. 12. $\cos \theta_{r}$ (squares) and $\cos \theta_{a}$ (circles) measured by Liu and $\operatorname{Kim}[33]$ vs $\cos \theta_{e}$ for "reentrant" structures with $\lambda \simeq 2.02, \alpha \simeq 1.12$, and $\epsilon \simeq 0.969$ calculated from their reported microscopic geometry. Filled and open symbols denote, respectively, a reentrant $\mathrm{SiO}_{2}$ surface coated with $\mathrm{C}_{4} \mathrm{~F}_{8}$ and a "doubly reentrant" one possessing a small overhang. For these periodic structures, $\cos \theta_{a}$ conforms to the superhydrophobic subset of regime $\mathrm{V}$ for $\cos \theta_{e} \lesssim 0.917$ (gray region). The solid line of slope $(1-\epsilon)$ and intercept $\epsilon$ is the corresponding prediction of $\cos \theta_{a}$ from Table II. Because regime $\mathrm{V}$ is metastable, its equilibrium receding angle (dotted line with $\cos \theta_{r} \lesssim 1$ ) is not readily achievable [23], and instead the observed $\cos \theta_{r}$ (squares) are nearer to $\cos \theta_{a}$. The transition from regime $\mathrm{V}$ to II occurs as $\chi_{c}^{+} \equiv 1$ [Table II and Eq. (24)] or, alternately, as $\cos \theta_{e}>$ $(\lambda-1) / \alpha \simeq 0.917$ (vertical dashed line). However, as $\cos \theta_{e}$ rises above this value, $\cos \theta_{a}$ loses equilibrium, thereby entering "regime II n.e.a." Nonetheless, because $\cos \theta_{r} \lesssim 1$ remains at equilibrium, Liu and Kim [33] observed an abrupt transition to $\cos \theta \sim 1$ as $\cos \theta_{e}$ rose above $(\lambda-1) / \alpha$ (solid circles, upper right corner). For its part, the "doubly reentrant" structure (open symbols) seemed to suppress this abrupt transition, perhaps as a serendipitous consequence of staging a surface with $(\lambda-1) / \alpha$ near 1 .

\section{CONCLUSIONS}

We presented a statistical mechanics predicting the equilibrium angles $\theta_{a}$ and $\theta_{r}$ of advance and recession of a liquid on a rough solid surface in terms of the Young contact angle $\theta_{e}$ on a flat plane of the same solid, and as functions of roughness geometry characterized by two parameters $\lambda$ and $\alpha$ representing, respectively, the specific area of cavities on the solid and the specific cross-section of necks joining them. We showed that contact angle behavior conforms to six possible regimes, including two with receding angle metastability.

The analysis indicated how the geometry of a bed of microscopic rods could be manipulated to create a wide range of contact angles and their hysteresis. The measurements of $\cos \theta_{a}$ by Onda, Shibuichi et al. [31,32] implied that several regimes could be explored with the same solid material by changing $\cos \theta_{e}$ through adjustments in liquid composition. In regimes I and II at low $\left|\cos \theta_{e}\right|$, their data suggested that $\cos \theta_{a}$ is a linear function of $\cos \theta_{e}$ with a noticeable intercept that our analysis attributed to interactions among neighboring cavities on the rough solid surface $(\lambda \neq 0)$. Their data and our analysis also implied the existence of a discontinuity in the linear relations of $\cos \theta_{a}$ versus $\cos \theta_{e}$ as the system moved from regime IV to regime II. A similar jump betwen regimes $\mathrm{V}$ and II was also evident in the data of Liu and Kim [33] for a superhydrophobic "reentrant" surface. 


\section{ACKNOWLEDGMENTS}

The author is grateful to David Quéré, Laurent Courbin, Alexandre Valance, Renaud Delannay, Jérome Crassous, Hervé Duval, Hervé Tabuteau, Andrew Clarke, Jin Xu, Shilpa Sahoo, Enrique Ramé, C.-Y. (Herbert) Hui, Olivier Desjardins,
Sheng Wang, Paul Steen, Jon Ludwicki, Tianshu Liu, and Mark Hurwitz for illuminating discussions, and to the anonymous reviewer for constructive suggestions. This paper was made possible by the support of NPRP Grant No. 6-059-2-023 from the Qatar National Research Fund and by National Science Foundation Grant No. CBET 1637531.
[1] R. E. Johnson and R. H. Dettre, J. Phys. Chem. 68, 1744 (1964).

[2] C. Priest, T. Albrecht, R. Sedev, and J. Ralston, Langmuir 25, 5655 (2009).

[3] M. A. Nilsson and J. P. Rothstein, J. Colloid Interface Sci. 363, 646 (2011).

[4] N. T. Chamakos, M. E. Kavousanakis, A. G. Boudouvis, and A. G. Papathanasiou, Phys. Fluids 28, 022105 (2016).

[5] G. Whyman, E. Bormashenko, and T. Stein, Chem. Phys. Lett. 450, 355 (2008).

[6] J. P. Rothstein, Annu. Rev. Fluid Mech. 42, 89 (2010).

[7] E. Wolfram, R. Faust, and J. F. Padday, Wetting, Spreading and Adhesion, edited by J. F. Padday (Academic, London, UK, 1978).

[8] E. Schäffer and P.-Z. Wong, Phys. Rev. E 61, 5257 (2000).

[9] D. Quéré, Annu. Rev. Mater. Res. 38, 71 (2008).

[10] D. Bonn, J. Eggers, J. Indekeu, J. Meunier, and E. Rolley, Rev. Mod. Phys. 81, 739 (2009).

[11] B. K. Larkin, J. Colloid Interface Sci. 23, 305 (1967).

[12] R. A. Brown, F. M. Orr, and L. E. Scriven, J. Colloid Interface Sci. 73, 76 (1980).

[13] Y. Rotenberg, L. Boruvka, and A. W. Neumann, J. Colloid Interface Sci. 102, 424 (1984).

[14] C. W. Extrand and A. N. Gent, J. Colloid Interface Sci. 138, 431 (1990).

[15] J. Xu and M. Y. Louge, Phys. Rev. E 92, 062405 (2015).

[16] J. D. Coninck, Colloids Surf., A 89, 109 (1994).

[17] R. Tadmor, Langmuir 20, 7659 (2004).

[18] H. Fujii and H. Nakae, Philos. Mag. A 72, 1505 (1995).

[19] W. G. Vincenti and C. H. Kruger, Introduction to Physical Gas Dynamics (Wiley, New York, 1965).

[20] J. B. Bostwick and P. H. Steen, J. Fluid Mech. 714, 336 (2013).
[21] L. Steub, J. Kollmer, D. Paxson, A. Sack, T. Pöschel, J. Bartlett, D. Berman, Y. Richardson, and M. Y. Louge, Microgravity spreading of water spheres on hydrophobic capillary plates, Powders and Grains, edited by F. Radjai (EDP Sciences, Les Ulis, France, 2017).

[22] A. B. D. Cassie and S. Baxter, Trans. Faraday Soc. 40, 546 (1944).

[23] A. Lafuma and D. Quéré, Nat. Mater. 2, 457 (2003).

[24] M. Callies and D. Quéré, Soft Matter 1, 55 (2005).

[25] L. Gao and T. J. McCarthy, Langmuir 22, 6234 (2006).

[26] E. Celia, T. Darmanin, E. T. de Givenchy, S. Amigoni, and F. Guittard, J. Colloid Interface Sci. 402, 1 (2013).

[27] S. Herminghaus, Europhys. Lett. 52, 165 (2000).

[28] M. Ma and R. M. Hill, Curr. Opin. Colloid Interface Sci. 11, 193 (2006).

[29] G. Whyman and E. Bormashenko, Langmuir 27, 8171 (2011).

[30] C. N. C. Lam, R. Wu, D. Li, M. L. Hair, and A. W. Neumann, Adv. Colloid Interface Sci. 96, 169 (2002).

[31] T. Onda, S. Shibuichi, N. Satoh, and K. Tsujii, Langmuir 12, 2125 (1996).

[32] S. Shibuichi, T. Onda, N. Satoh, and K. Tsujii, J. Phys. Chem. 100, 19512 (1996).

[33] T. L. Liu and C.-J. Kim, Science 346, 1096 (2014).

[34] A. Tuteja, W. Choi, J. M. Mabry., G. H. McKinley, and R. E. Cohen, Proc. Natl. Acad. Sci. USA 105, 18200 (2008).

[35] R. N. Wenzel, Ind. Eng. Chem. 28, 988 (1936).

[36] Z. Yoshimitsu, A. Nakajima, T. Watanabe, and K. Hashimoto, Langmuir 18, 5818 (2002).

[37] F. Mugele and J.-C. Baret, J. Phys.: Condens. Matter 17, R705 (2005).

[38] W. C. Nelson and C.-J. Kim, J. Adhesion Sci. Tech. 26, 1747 (2012). 